\title{
Measurement of Economic Linkage Different Development of Urban Agglomeration
}

\author{
Lei Li' ${ }^{1}$, Weiqi Guo ${ }^{2}$, Zhanbo Chen ${ }^{2}$ \\ ${ }^{1}$ Central China Normal University, Wuhan, China \\ ${ }^{2}$ Zhongnan University of Economics and Law, Wuhan, China \\ Email: hbuewqy@qq.com
}

How to cite this paper: Li, L., Guo, W.Q. and Chen, Z.B. (2018) Measurement of Economic Linkage Different Development of Urban Agglomeration. Open Access Library Journal, 5: e4453.

https://doi.org/10.4236/oalib.1104453

Received: February 27, 2018

Accepted: March 24, 2018

Published: March 27, 2018

Copyright ( $\odot 2018$ by authors and Open Access Library Inc.

This work is licensed under the Creative Commons Attribution International License (CC BY 4.0).

http://creativecommons.org/licenses/by/4.0/

c) (7) Open Access

\begin{abstract}
At present, the economic development in Hubei Province has initially entered new era of "one principal two deputy, multi-polar development". In order to further explore the future of urban development, we ranked prefecture cities in Hubei Provinces with the intensity model of central place function. Meanwhile, according to 32 selected indicators, we investigated the economic linkage between these cities with improved gravity model and principal component analysis model. Our results show that: 1) Regarding intensity value of central city $\mathrm{z}=2,0.9,0.6$ as the boundary, 12 cities can be divided into four levels with Wuhan in level 1; Yichang, Xiangyang and Jingzhou in level 2; Huangshi, Shiyan, Jingmen, Xiaogan, Huanggang, Xianning in level 3; the remaining cities in level 4, respectively. 2) According to the economic linkage value, the economic linkages between cities are classified into three levels: closely related, generally related and weakly related. Among them, the generally related and weakly related linkages are most likely caused by the cities' own economic development structure or their long geographical distance from the economic central city.
\end{abstract}

\section{Subject Areas \\ Information Science}

\section{Keywords}

Gravity Model, Intensity of Cental Place Function, Economic Linkage

\section{Introduction}

In recent years, along with the continuous development of economic globalization, the mode of regional economic integration and urban agglomeration has 
increasingly become the mainstream in various regions of the world. Through these modes, cities become more competitive in the global economic competition and specialization. The prominent representatives like the European Union, the North American Free Trade Area, the urban agglomeration of the Atlantic coast in the northeastern United States and the urban agglomeration of the Pacific coast of Japan have not only accelerated the circulation of various resources within their regions, promoted the optimization of industrial structure, but also further strengthened the significance of the regions in the global economic landscape.

Hubei Province is located in the "waist of the Yangtze River" and plays a decisive role in the construction of urban agglomerations in the mid-Yangtze. Moreover, as the only mega-city in the central region, Wuhan, the capital of Hubei Province, its economic linkages with other cities in the province play a decisive role in the economic development of Hubei Province and will have a huge impact on the economic development of urban agglomerations in the mid-Yangtze. It can be concluded that at present, China implements the strategy of the Yangtze River Economic Belt and vigorously promotes the urban agglomeration in the mid-Yangtze, which is the most precious opportunity for the future development of Hubei province. Hubei should seize the opportunity, take Wuhan as the core competence to promote the economic development coordination of all the cities and further strengthen the economic linkages with each other, so as to enhance the competitiveness and importance of Hubei province in the national even the international economic environment.

\section{Literature Review}

The essence of urban economic linkage is to promote the economic links between cities through the mutual circulation of resources such as people, things and information between cities. The measurement of economic linkages between cities is to measure the closeness of economic linkages between cities through various quantifiable indicators. The related research of regional economic linkages has been a hot research topic in the western countries which have developed urban agglomeration. Starting from using gravitation models to do quantitative analysis of regional spatial linkages (WJ Reilly, 1929), foreign experts and scholars continuously explore quantitative analysis of regional spatial linkages, making the theory of urban economic linkages measurement theory continuously enriched and improved. After WJ Reilly, some scholars applied the law of universal gravitation to the study of urban agglomeration (GK Zipf, 1942) or used the theory of break point to calculate the interaction between city and region based on gravitational model (PD Converse, 1949). Some applied the input-output measurement model to measure the regional economic spatial linkage (Walter Isard, 1951). The measurement scope is not only limited to the regional spatial economic linkage, but also gradually expands to the aspect of city's functional linkage and the outward service. 
Domestic research on urban economic relations started later, and initial research was limited to qualitative analysis. Until 1990, the mathematical measurement model was applied. For the specific model, the current domestic scholars mainly use the gravity model proposed by WJ Reilly or improve the gravity model and interval input-output model, or use social network analysis, geo-economic linkage matching analysis and other methods to measure. And because the reform and opening-up policy of China is a process from the coast to the inland as well as from the point to the surface, the urban agglomerations in the eastern coastal areas are more mature and their economic linkages are closer. Therefore, studies of most of the scholars are related to area of eastern coast. Le Zhang, Yongjun Ma (2013), Xiangjun Ou and Liping Xue et al. (2015) respectively analyzed the spatial linkage of urban space in Zhejiang and Jiangsu Provinces by using gravity model and social network model. They analyzed the economic linkage direction, link intensity and the differences between the central cities, pointed out the direction of economic development in the two provinces [1]. Scholars such as Jinping $\mathrm{Lu}$ and Zhenwu Yang (2015) used the intensity model of central place function to classify the Beijing-Tianjin-Hebei urban agglomeration, and then used the improved gravitation model to measure the urban economic linkage. Finally, it explains the development differences in the integration process of Beijing-Tianjin-Hebei urban agglomeration from three aspects: diffusion of urban agglomeration, natural resource endowment and location advantages [2]. Lin Zou, Gang Zeng et al. (2015) took the Yangtze River economic belt as the research content, used social network analysis and nuclear density estimation based on the economic network model. They empirically analyzed the economic network structure, central nodes, network density and network subgroup characteristics of the Yangtze River economic belt, and proposed regional optimization suggestions for accelerating the coordinated development of the economic belt [3]. Fei Gong and Guansheng Lv (2015) measured the economic linkages between the four central cities of North Gulf in Guangxi Province from the perspective of tourism economy of developing urban agglomeration. The calculation shows that the tourism economic development in the above regions is still at a low level and regional cooperation needs to be further strengthened, thus to promote further development of the regional economy [4].

Based on the previous studies, this paper takes 12 prefecture-level cities in Hubei province as the research content. First of all, takes the index system developed by the Urban Division of National Bureau of Statistics and the research group of urban statistics of China statistical society as reference. Secondly, establishes the index system of urban economic linkage measurement based on the evaluation indicators adopted by the above scholars, considering the quantifiability and the availability of index. Then, with the help of the intensity model of central place function, combines grading method by natural crack point to classify the city. After that, uses the principal component analysis model to evaluate the comprehensive economic intensity of 12 cities and sort them according to their scores. Finally, this paper analyzes and estimates the intensity of the eco- 
nomic linkages between the 12 cities in Hubei Province and provides some suggestions for the future construction, planning and development policy of the urban agglomeration, which will further promote the urban development in the mid-Yangtze.

\section{Establishment of Evaluation Index System of Urban Economic Comprehensive Strength}

\subsection{Data Resource}

In order to quantify and evaluate the economic comprehensive economic intensity of the city, the corresponding index system must be established in accordance with the principle of completeness and science. Based on the existing index system, this paper chooses a comprehensive index system of urban economic development including human resources, economic resources, social development resources, infrastructure resources and ecological protection resources.

Human resources are the most active and valuable resources for urban development. People are both the main body of social wealth and the main driving force for maintaining social progress. The population as well as the quality and structure of the population will have far-reaching and direct influence for a city. Therefore, five indicators are selected for human resources: Year-end Population, natural population growth rate, the proportion of registered unemployed population in the total population in urban areas, the proportion of tertiary industry employees in the total population, the population density.

Economic resources are important aspects that directly reflect the current strength of cities as well as the closeness of spatial relationships with other cities. The current economic scale and economic structure will have a significant impact on the overall economic development. However, the selection of indicators of economic resources should not only include the stock indicator but also the incremental indicator. It is necessary to reflect not only the activity level of the internal economy but also the external economy linkage. Therefore, we should select 10 indicators of economic resources including: GDP, The ratio of tertiary industry and GDP, The balance of deposits and loans of financial institutions at the end of the year, Total industrial output value, Total volume of tourist transport, Total freight volume, Business income of postal, Total retail sales of social consumer goods, The actual amount of foreign investment, The social development resources. The economic development of a city can not only pursue short-term development but also the long-term development, so that the city can achieve sustainable economic development. However the indicators that support the sustainable development of urban economy are often the implicit indicators that indirectly promote the current economic development such as science and education. Therefore, we should select 8 social development resources: fiscal revenue, science and technology expenditure, education expenditure, number of Internet broadband users, number of college students per 10,000 people, the public library collection per hundred people, number of hospitals and clinics, the 
number of people participating in urban basic medical insurance.

Infrastructure construction not only provides more superior hardware conditions and external environment for the development of urban economy, but also is an important aspect of a city in attracting foreign talents. Furthermore, it relates to the direct interests of the residents living condition in the city. Therefore, eight indicators are selected for the infrastructure resources: the length of drainage pipes, the total water supply, the total social electricity consumption, the number of cars owned by each 10,000 people, the urban road area per capita.

The ecological protection resource is the basis for maintaining a city, it provides the most basic place and material resources. Good ecological system is the most fundamental guarantee for the sustainable development of a city. In the process of urban construction and development, we should not only pay attention to the indicators that reduce the damage to the ecological environment, but also focus on the indicators that are conducive to improve the ecological system. Therefore, four indicators are selected for ecological protection resources: green coverage rate of built-up area, utilization of general industrial solid waste, centralized treatment rate of sewage treatment plant, harmless treatment of household garbage [5]. To sum up, the paper establishes an indicator system of urban economic relations that includes 32 specific indicators in five major development areas. The specific indicators are shown in Table 1.

\subsection{Data Selection and Pre-Processing}

As the 32 indicators belong to 5 different aspects, the dimensions of the indicators are not uniform, and their promotion effects on urban development are also different, so the indicators need to be treated in a non-dimensional method. For the conventional positive and negative indicators, this paper standardize them by using the extreme value, it can be seen in the formula 1 :

$$
\left\{\begin{array}{l}
x_{i}=\left(x_{i}-\min x_{i}\right) /\left(\max x_{i}-\min x_{i}\right) \text { positive index } \\
x_{i}=\max x_{i}-x_{i} / \max x_{i}-\min x_{i} \text { negative index }
\end{array}\right.
$$

where $x_{i}$ is the value of index $i$. However, for the neutral indexes like natural population growth rate and the population density, obviously, it is not it is impossible to standardize them directly by using the above formula. So they need to be transformed respectively. Examining the authoritative information we know that: at present, in order to maintain the sustainable development of the population in our country, under the precondition of opening the two-child per family policy, the natural growth rate of our country's population should be maintained at about 5.5\%. Meanwhile, for the population density, the common international standard 500 people $/ \mathrm{km}^{2}$ is relatively reasonable. Therefore, the method of dealing with the neutral index in this paper is as follows: Taking the common policy as the optimal standard, calculate the absolute value of the difference between each index of the 12 cities and the optimal standard. The smaller the absolute value of the difference is, the better the value is. Therefore, 
Table 1. Comprehensive evaluation index system of urban economy.

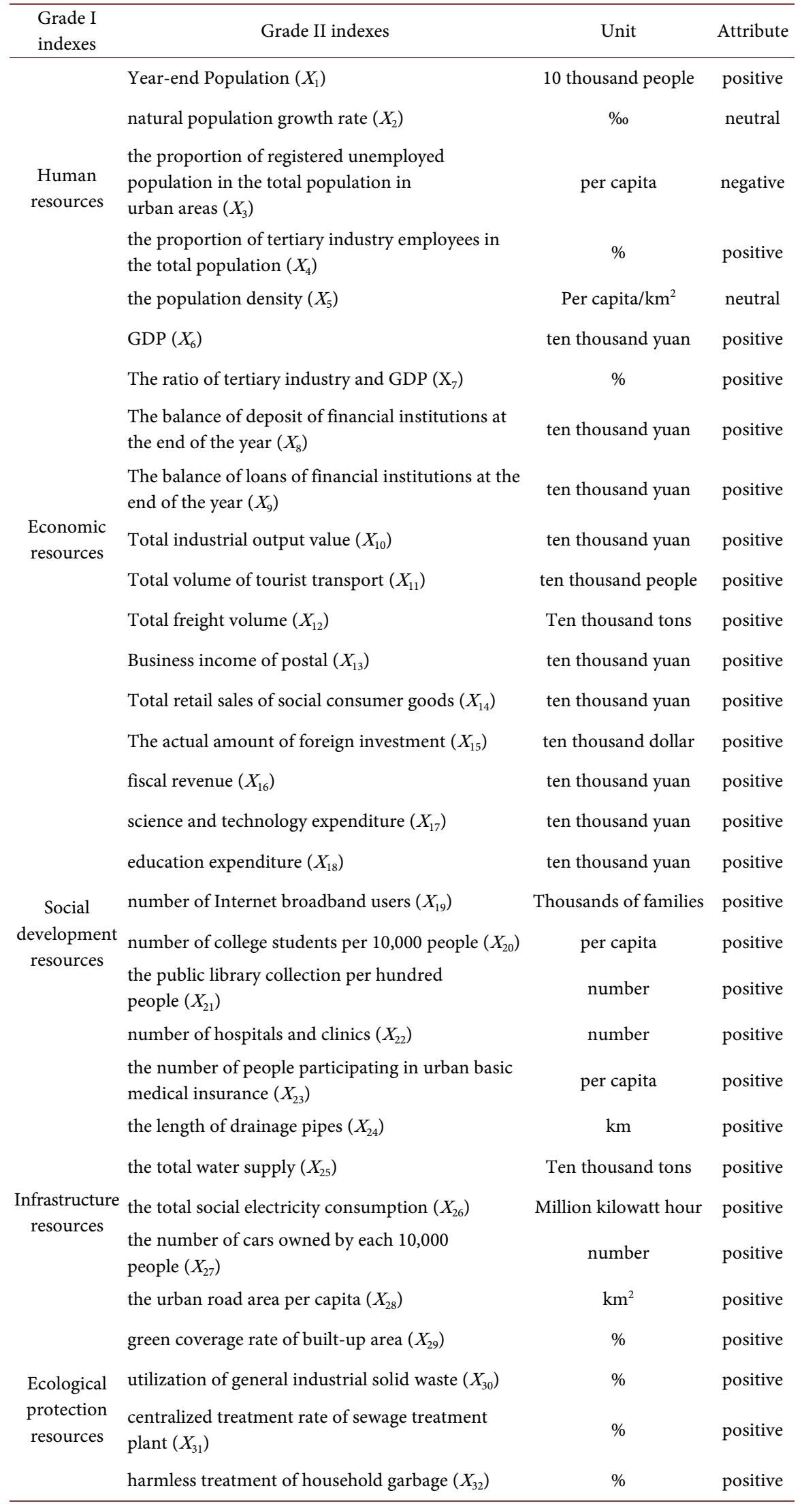


we can convert a neutral index into a negative index.

At the same time, for some of the disputed indicators such as the proportion of tertiary industry employees in the total population, the ratio of tertiary industry and GDP, the urban road area per capita, they can not be fully included within the scope of the positive indicators in theory, because the tertiary industry represents the economic prosperity of a country or region, but excessive prosperity will bring enormous devastation to the real economy such as the primary and secondary industries. In the same way, the urban road area per capita can not be as large as possible. According to the actual development of 12 cities, the above three indexes have not reached the average level of the developed countries in the world (the ratio of tertiary industry and GDP in developed countries is higher than $60 \%$ and the urban road area per capita is 30 square meters). Therefore, they are still classified as a positive indicator in this paper for standardization.

For the authenticity and scientific of data, all the data in this evaluation index system are selected from "Statistical Yearbook of Hubei Province in 2015", "Statistical Yearbook of China's Cities in 2015" and Official Website of Hubei Bureau of Statistics.

\section{Application of Intensity Model-Classification of Cities}

The development of regional economy will inevitably have differentiated characteristics under the combined influence of innate factors such as natural resources and transportation location and acquired factors such as state policies and population movements. Because of the different social division and the different industrial structures, cities in the same area will have different roles in the process of economic development. Often, there will be a batch of cities with integrated and pivotal functions to coordinate and dominance the development of regional economy and promote the common development of cities and regional economies. A city with this kind of function is regarded as a central city in the development of regional economy.

At present, most of the regional central cities in our country are provincial capitals or cities with special development advantages. However, the functions undertaken by other cities in the region are often ignored by researchers. Therefore, this article will introduce the intensity model of central place function to measure the prefecture cities in Hubei Province. This theory was originally put forward by German economic geographer Chris Taylor in the early 1930s. It was measured according to the telephone index at the beginning. The principle is based on the selection of evaluation indicators, by calculating the proportion of city's index and the average value of such index of all cities, obtain the functional index of the city. Lastly, all functional indexes are averaged to obtain the city's central functional strength. After that, scholars in various fields continued to expand and improve the indexes, so they can use different indexes or comprehensive indexes for measurement. 
Based on the previous studies, this paper applies the intensity model of central function to measure the urban centrality of 12 cities in Hubei province. Therefore, select seven indexes to calculate the index $\mathrm{N}$ of city function: Year-end Population $\left(n_{1}\right)$, the proportion of tertiary industry employees in the total population $\left(n_{2}\right)$, GDP $\left(n_{3}\right)$, Total retail sales of social consumer goods $\left(n_{4}\right)$, number of college students per 10,000 people $\left(n_{5}\right)$, the total social electricity consumption $\left(n_{6}\right)$, green coverage rate of built-up area $\left(n_{7}\right)$. The calculation method is shown in Equation (1):

$$
N_{i j}=\frac{n_{j}}{\frac{1}{n} \sum_{j=1}^{n} n_{j}} ; \quad(i=1,2, \cdots 12 ; j=1,2, \cdots 7)
$$

Sum up the number of functions and calculate the arithmetic average value of each city, that is the intensity value $\mathrm{Z}$ of central function, as shown in Equation (2):

$$
Z_{N_{i j}}=\frac{N_{i 1}+N_{i 2}+\cdots+N_{i j}}{j} ; \quad(i=1,2, \cdots 12 ; j=1,2, \cdots 7)
$$

According to formulas (1) and (2), we can calculate the intensity value of 12 prefecture cities in Hubei Province. The evaluation principle is: As each indicator is a positive indicator, so in this paper, the value of the central function is also a positive value. That is, the larger the value is, the more city functions are undertaken by the city in the province and the more prominent the center is. At the same time, the city is divided into four levels according to the natural crack classification method according to the specific intensity value of each city (if $Z_{N^{i j}}>2$, it is first-level central city; if $0.9<Z_{N^{i j}} \leq 2$, it is second-level central city; if $0.6<Z_{N^{i j}} \leq 0.9$, it is third-level central city; if $Z_{N^{i j}} \leq 0.6$, it is forth-level central city), The specific results is shown in Table 2.

The functional intensity and hierarchy of 12 prefecture cities in Hubei Province are shown in Table 2. It can be concluded from Table 2:

1) Due to its unique geographical location and political advantages, Wuhan plays an absolutely central leading role in the province. Its value is much higher than other cities, indicating that the development of Wuhan has strong economic radiation and is dominant in the process of economic development in Hubei Province;

2) Wuhan has the highest intensity value, followed closely by Yichang, Xiangyang and Jingzhou. Although there is still a big gap between wuhan and these three cities, they also have a distinct central advantages and the driving force compared with other cities, indicating that these three cities have important positions that can not be ignored in the urban development process.

3) Compared with the cities of first two levels, the intensity of central place function in Huangshi, Shiyan, Jingmen, Xiaogan, Huanggang and Xianning is not dominant, but each city has its unique development path. They have their own characteristics during the regional economic development, but the 
Table 2. Central city classification of 12 prefecture cities in Hubei Province.

\begin{tabular}{|c|c|c|c|c|c|c|c|c|c|c|c|c|}
\hline & Wuhan & Huang shi & Shiyan & Yichanga & Xiangyang & Ezhou & Jingmen & Xiaogan & Jingzhou & Huanggang & Xianning & Suizhou \\
\hline$n_{1}$ & 1.864 & 0.597 & 0.782 & 0.902 & 1.342 & 0.248 & 0.677 & 1.185 & 1.484 & 1.671 & 0.668 & 0.579 \\
\hline$n_{2}$ & 1.096 & 0.837 & 1.100 & 0.996 & 0.851 & 0.722 & 0.969 & 0.919 & 1.144 & 0.868 & 1.336 & 1.162 \\
\hline$n_{3}$ & 4.521 & 0.547 & 0.539 & 1.406 & 1.405 & 0.308 & 0.588 & 0.608 & 0.665 & 0.655 & 0.433 & 0.325 \\
\hline$n_{4}$ & 4.738 & 0.563 & 0.595 & 1.046 & 1.117 & 0.250 & 0.490 & 0.747 & 0.902 & 0.776 & 0.392 & 0.384 \\
\hline$n_{5}$ & 5.937 & 0.711 & 0.731 & 0.755 & 0.451 & 0.686 & 0.347 & 0.381 & 0.867 & 0.320 & 0.692 & 0.123 \\
\hline$n_{6}$ & 5.512 & 0.957 & 0.602 & 1.076 & 0.891 & 0.940 & 0.595 & 0.270 & 0.618 & 0.135 & 0.234 & 0.172 \\
\hline$n_{7}$ & 1.116 & 0.918 & 1.032 & 1.178 & 0.913 & 0.924 & 1.138 & 0.629 & 1.114 & 0.731 & 1.066 & 1.242 \\
\hline$Z n$ & 3.540 & 0.733 & 0.769 & 1.051 & 0.996 & 0.583 & 0.686 & 0.677 & 0.970 & 0.736 & 0.689 & 0.570 \\
\hline Level & first & third & third & second & second & forth & third & third & second & third & third & forth \\
\hline
\end{tabular}

economic radiation and driving capability of the surrounding areas are relatively weak.

4) Ezhou and Suizhou due to its lower $Z_{N^{i j}}$ value, the function of central place is weak and the driving effect on the regional economy is not obvious. The economic influence in the whole province is also relatively small.

\section{Comprehensive Evaluation of Urban Economy Development and Measurement of Economic Linkages}

\subsection{Evaluation of Overall Strength of Urban Economy Development}

The calculation of the economic connection between cities must be based on the comprehensive economic strength of each city. When we select the index of comprehensive evaluation system of urban economy, considering the comprehensiveness of index selection, some indicators have the problem of information redundancy. In order to reduce information redundancy of the indexes, use the principal component analysis and the evaluation system to calculate the comprehensive economic strength value, because principal component analysis can reorganize the initial correlated variables into a group of irrelevant indicators, and then reduce the relevance of the indicators as well as the dimension. The result is shown in Table 3.

From Table 4, we can see that in accordance with the principle that the initial eigenvalue is greater than 1, 5 new principal component factors are selected to replace the original 32 indicators to evaluate the comprehensive economic strength of the city. Moreover, from the cumulative contribution rate of variance, it can be seen that the contribution rate of the five principal component factors is as high as $92.511 \%$ (far greater than the standard of $85 \%$ ), indicating that the five principal component factors contain a large amount of information of the original indicator. The comprehensive economic strength can be well evaluated. 
Table 3. Conclusion of principal component analysis.

\begin{tabular}{ccccccc}
\hline \multirow{2}{*}{ Component } & \multicolumn{3}{c}{ Initial Eigenvalues } & \multicolumn{3}{c}{ Rotated Square Sum Load } \\
\cline { 2 - 7 } & Sum & Variance\% & Accumulation \% & Sum & Variance \% & Accumulation \% \\
\hline 1 & 21.066 & 65.83 & 65.83 & 20.095 & 62.796 & 62.796 \\
2 & 3.361 & 10.503 & 76.333 & 3.582 & 11.192 & 73.988 \\
3 & 2.373 & 7.417 & 83.75 & 2.372 & 7.414 & 81.402 \\
4 & 1.769 & 5.527 & 89.277 & 1.996 & 6.238 & 87.64 \\
5 & 1.035 & 3.234 & 92.511 & 1.559 & 4.871 & 92.511 \\
\hline
\end{tabular}

Table 4. The comprehensive economic score of 12 cities in Hubei Province.

\begin{tabular}{cccccc}
\hline Wuhan & Huangshi & Shiyan & Yichang & Xiangyang & Ezhou \\
\hline 14.139 & 1.222 & 1.547 & 2.543 & 2.522 & 0.213 \\
Jingmen & Xiaogan & Jingzhou & Huanggang & Xianning & Suizhou \\
1.096 & 0.941 & 1.629 & 1.751 & 0.661 & 0.889 \\
\hline
\end{tabular}

Finally, taking the variance contribution of each principal component factor as a coefficient (As shown in Equation (3)), calculate the economic comprehensive strength of each city, and the result is shown in Table 4:

$$
Y=0.6583 t_{1}+0.10503 t_{2}+0.07417 t_{3}+0.05527 t_{4}+0.03234 t_{5}
$$

where $Y$ is the comprehensive score of the final economic strength of each city, $t_{1}, t_{2}, t_{3}, t_{4}$ and $t_{5}$ are the factor loadings of five principal components respectively.

\subsection{Measurement of Urban Economic Linkages}

In order to measure the economic linkages between cities, this paper introduces the gravitation model of the predecessor [6]. The calculation method is shown in formula (4):

$$
R_{i j}=\left(\sqrt{p_{i} * v_{i}} \times \sqrt{p_{j} * v_{j}}\right) / D_{i j}^{2}
$$

where $R_{i j}$ denotes the economic attractiveness of city $i$ to city $j, p_{i}$ and $p_{j}$ denote the population owned by each city, $v_{i}$ and $v_{j}$ denote the comprehensive strength of each city's economic development, and $D_{i j}$ denotes the geographical distance between the two cities.

The formula is derived from Newton's formula of universal gravitation and can be used to measure the economic linkage between the two regions. However, since the above formula do not consider the situation that there is a big difference between the development bases and development conditions of cities, the formulas need to be amended. Therefore, when we calculate the economic linkage between the two cities, appropriate weigh $k_{i j} k_{i j}=v_{i} /\left(v_{i}+v_{j}\right)$ must be given. Taking the respective economic comprehensive strengths of the two cities as their weights, the actual economic linkage between the two cities can be better 
reflected. Because each index in the formula is converted into non-dimensional variable, the meaning represented by the numerical values of each index only indicates the degree of superiority and inferiority, not the optimal value of the actual distance, and non-dimensional variable can be compared horizontally and vertically. Therefore, the economic connection between cities can be calculated. Finally, the revised method of calculating economic linkages value is shown in formula (5):

$$
R_{i j}=k_{i j}\left(\sqrt{p_{i} * v_{i}} \times \sqrt{p_{j} * v_{j}}\right) / D_{i j}^{2}, \quad k_{i j}=v_{i} /\left(v_{i}+v_{j}\right)
$$

Finally, take the original data of the total population at the end of each year, the score of economic comprehensive strength and the actual geographical distance of the cities (the geographical distance between cities takes the linear distance between Municipal Governments as the standard) into formula (5). Calculate the economic linkages between 12 prefecture cities in Hubei Province, the economic linkage value is consistent with the evaluation principle of the functional strength of the city center that the economic linkage value between cities is also a positive indicator, while the larger the value is, the closer the economic linkage between the two cities is the specific results are shown in Table 5:

It can be seen from Table 5 that there is a great difference between the economic linkages among cities. In order to facilitate the analysis, the degree of economic linkage is divided into 3 levels:

1) If the economic linkage value $\geq 10$, the cities are closely related. Like $\mathrm{Wu}$ han $\rightarrow$ Yellowstone, Wuhan $\rightarrow$ Yichang, Wuhan $\rightarrow$ Xiangyang, Wuhan $\rightarrow$ Xiaogan, Wuhan $\rightarrow$ Jingzhou, Wuhan $\rightarrow$ Huanggang, Wuhan $\rightarrow$ Xianning, Wuhan $\rightarrow$ Suizhou, Huanggang $\rightarrow$ Huangshi and Huanggang $\rightarrow$ Ezhou. Wuhan, as the capital city of Hubei province, has all kinds of advantageous resources concentrated and has strong economic radiation function to other cities in the province. Therefore, wuhan has close economic linkage with the rest of the cities. In addition, the geographical space among Huanggang, Huangshi and Ezhou is relatively close, it is easy to form a complementarity of the industries, so the economic linkage between Huanggang and the other two cities are also very close;

2 ) If $3 \leq$ the economic linkage value economic contact value $<10$, the cities are generally related. Like Wuhan $\rightarrow$ Shiyan, Wuhan $\rightarrow$ Ezhou, Wuhan $\rightarrow$ Jingmen, Huangshi $\rightarrow$ Ezhou, Huangshi $\rightarrow$ Huanggang, Yichang $\rightarrow$ Xiangyang, Yichang $\rightarrow$ Jingmen, Yichang $\rightarrow$ Jingzhou, Xiangyang $\rightarrow$ Shiyan, Xiangyang $\rightarrow$ Jingmen, Xiangyang $\rightarrow$ Xiaogan, Xiangyang $\rightarrow$ Jingzhou, Xiangyang $\rightarrow$ Huanggang, Xiangyang $\rightarrow$ Suizhou, Ezhou $\rightarrow$ Huanggang, Xiaogan $\rightarrow$ Wuhan, Xiaogan $\rightarrow$ Huanggang, Jingzhou $\rightarrow$ Jinmen, Jinzhou $\rightarrow$ Xiaogan, Huanggang $\rightarrow$ Wuhan, Huanggang $\rightarrow$ Xianning. Such economic linkages are relatively loose. Yichang and Xiangyang are the center of these linkage, Jingzhou and Jingmen act as auxiliaries. The economic radiation range is also more concentrated in mid-west or north-west of Hubei Province. It promoted the economic development by the way of point to area. 
Table 5. The economic linkage values of the 12 prefecture cities in Hubei province.

\begin{tabular}{ccccccccccccc}
\hline & Wuhan & Huangshi & Shiyan & Yichang Xiangyang & Ezhou & Jingmen & Xiaogan & Jingzhou & Huanggang & Xianning & Suizhou \\
\hline Wuhan & 0 & 22 & 6 & 10 & 14 & 9 & 9 & 46 & 16 & 61 & 17 & 10 \\
Huangshi & 2 & 0 & 0 & 1 & 1 & 3 & 1 & 2 & 1 & 8 & 2 & 1 \\
Shiyan & 1 & 0 & 0 & 1 & 2 & 0 & 1 & 1 & 1 & 1 & 0 & 1 \\
Yichang & 2 & 1 & 2 & 0 & 4 & 0 & 4 & 2 & 6 & 2 & 1 & 2 \\
Xiangyang & 2 & 1 & 4 & 4 & 0 & 1 & 5 & 3 & 4 & 3 & 1 & 3 \\
Ezhou & 0 & 1 & 0 & 0 & 0 & 0 & 0 & 0 & 0 & 3 & 0 & 0 \\
Jingmen & 1 & 1 & 1 & 2 & 2 & 0 & 0 & 1 & 3 & 1 & 1 & 1 \\
Xiaogan & 3 & 1 & 1 & 1 & 1 & 1 & 1 & 0 & 2 & 3 & 1 & 2 \\
Jingzhou & 2 & 1 & 1 & 4 & 3 & 1 & 5 & 3 & 0 & 2 & 2 \\
Huanggang & 8 & 11 & 1 & 1 & 2 & 21 & 2 & 5 & 2 & 0 & 4 & 2 \\
Xianning & 1 & 1 & 0 & 0 & 0 & 1 & 0 & 1 & 1 & 2 & 0 \\
Suizhou & 1 & 1 & 0 & 1 & 1 & 0 & 1 & 2 & 1 & 1 & 1 \\
\hline
\end{tabular}

3) If the economic linkage value $<3$, the cities are weakly related, most of the linkages except the two levels above are in this category. Owing to their relatively simple economic structure and relatively far distance from the economic central city and the regional central cities, their economic links with other cities are also sparse and loose. For example, Xianning, as the southern gate of Hubei Province, is relatively far from Wuhan compared with the distance between Wuhan and Huangshi, Wuhan and Huanggang, Wuhan and Ezhou. There are also some restrictions due to its own geographical conditions like mountainous and hilly make Xianning less related to other cities.

\section{Conclusion}

The economic development of cities in the coastal provinces of China is relatively balanced due to the support of the state policy and its exploration development mode from point to area. However, in the central and western inland provinces, political, economic, cultural and other social resources are highly concentrated in capital cities due to historical traditions. This has often results in the monopoly of a city in one province. Through the above research, we can see that in the economic development of Hubei Province, Wuhan not only is the central city, but also maintains strong economic linkages with the other cities in the province. Exhausting the power of the whole province to build capital cities is not only detrimental to the sustainable development of the regional economy but also undermines the economic development of other cities in the province. Therefore, it is necessary to scientifically formulate plans for economic development, coordinate the economic development among cities and make the economic development of cities in the provinces have their unique path and characteristics. Only coordinated development of all the cities, can we promote the 
further development of Hubei Province, and then promote the growth of the regional economy in Central China.

\section{Acknowledgements}

This work was supported by the China Key projects of the National Bureau of Statistics under Contracts 2016564. The authors would also like to thank the anonymous reviewers for their useful comments.

\section{References}

[1] Xue, L.P., Ou, X.J., Zeng, C. and Qiao, S.S. (2014) Analysis on the Spatial Function of Economic Linkage between Major Cities in Huaihai Economic Zone. Economic Geography, 11, 52-57.

[2] Lu, J.P., Yang, Z.W. and Sun, J.W. (2015) Research on the Economic Linkage Measurement of Beijing-Tianjin-Hebei Urban Agglomeration. Urban Studies, 1, 5-10.

[3] Zou, L., Zeng, G., Cao, X.Z. and Chen, S.Y. (2015) Analysis on the Spatial Features of Economic Linkage in the Yangtze River Economic Belt. Economic Geography, 6 , $1-7$.

[4] Gong, F. and Lv, G.S. (2015) Measurement and Evaluation of Tourism Economic Linkage Degree of North Gulf of Guangxi District's Central City. East China Economic Management, 5, 75-78.

[5] Wang, Q.Y., Dai, H.N. and Wang, H. (2017) A Smart MCDM Framework to Evaluate the Impact of Air Pollution on City Sustainability: A Case Study from China. Sustainability, 9, 911. https://doi.org/10.3390/su9060911

[6] Gao, X.L. and Yin, W.H. (2015) The Evolution of Urban Economic Linkage in Shandong Province Based on Gravity Model. China Population Resources and Environment, 25, 458-461. 